Théologiques

Théologiques

\title{
Fide splendet et scientia
}

Du sens d'une devise

\section{Jean Grondin}

Volume 13, numéro 1, printemps 2005

Croire et croyances

URI : https://id.erudit.org/iderudit/012528ar

DOI : https://doi.org/10.7202/012528ar

Aller au sommaire du numéro

\section{Éditeur(s)}

Faculté de théologie et de sciences des religions, Université de Montréal

ISSN

1188-7109 (imprimé)

1492-1413 (numérique)

Découvrir la revue

Citer cet article

Grondin, J. (2005). Fide splendet et scientia : du sens d'une devise. Théologiques, 13(1), 119-127. https://doi.org/10.7202/012528ar
Résumé de l'article

En partant d'une réflexion sur la devise de l'Université de Montréal, «C'est par la foi et la science qu'elle resplendit ", cet article se penche sur l'altérité fondamentale de la foi par rapport à la science, voire sur sa priorité. Il se demande si la science, dans la mesure où elle doit se tenir à une certaine distance de son objet, est en mesure de comprendre quelque chose à l'expérience de la foi. À la différence de la science, la foi est peut-être moins une forme de maîtrise que de dépossession, moins un savoir qu'un non-savoir.
Tous droits réservés @ $\odot$ Faculté de théologie et de sciences des religions, Université de Montréal, 2006
Ce document est protégé par la loi sur le droit d'auteur. L'utilisation des services d'Érudit (y compris la reproduction) est assujettie à sa politique d'utilisation que vous pouvez consulter en ligne.

https://apropos.erudit.org/fr/usagers/politique-dutilisation/ 


\section{Fide splendet et scientia}

\section{Du sens d'une devise ${ }^{1}$}

Jean GRONDIN

Département de philosophie Université de Montréal

Ce numéro de la revue Théologiques porte sur la difficile question du croire et des croyances. Mais peut-on parler du croire et des croyances comme de données qui pourraient se dresser devant nous, comme on peut parler, par exemple, des quartz, des dauphins, de l'œuvre de Racine ou de l'histoire du $\mathrm{XIX}^{\mathrm{e}}$ siècle? Autrement demandé: peut-on parler du croire sans s'engager soi-même? Il y a là tout un défi, qui a tout à voir avec une question, que je ne suis évidemment pas le premier à poser: peut-on vraiment comprendre quelque chose au croire, à la croyance ou à la foi à partir de la perspective du savoir? Dans la mesure où elle se tient à une certaine distance de son objet, la science est-elle en mesure de comprendre quelque chose à l'expérience de la foi ? N'est-elle pas, cette expérience, quelque chose qui se refuse, et de manière essentielle, à une saisie extérieure ou, pire, constructive? Et déjà, parler de l' "expérience» de la foi, n'est-ce pas emprunter un vocabulaire qui n'est pas le sien? Qu'est-ce que la foi, au juste ? Une "expérience », un "phénomène », une «illusion », un acte ou, au contraire, une passion de l'intelligence, voire de la volonté ? Dans toutes ces acceptions, la foi est un peu comprise comme quelque chose qui dépend du «sujet». Or, ce n'est pas toujours de cette manière qu'elle a été éprouvée. Souvent, elle a plutôt été comprise comme une «soumission» de l'homme à quelque chose qu'il ne peut pas comprendre. Cette idée vient, bien entendu, de la Lettre aux Romains $(1,5)$, qui parle ici de l'hupakoè pisteôs, de l'obéissance, sinon de la soumission qu'est la foi, et d'une obéissance qui est affaire d'écoute (on entend, bien sûr, l' " acoustique », du verbe akouô, dans l'hupakoè, selon une assonance qui se retrouve un peu en allemand dans la correspondance entre le

1. Texte présenté dans le cadre du colloque « Religion : croire et croyances » (Montréal, 6 février 2003) organisé par le CÉRUM. 
gehorchen et le horchen - il est bien connu que le terme «islam» a aussi le sens d'une soumission. Et cette écoute est, bien entendu (!), celle d'une voix qui me précède (voir Rm 10,17 au sujet de la foi qui vient de l'écoute des choses qui nous ont été dites, voire d'un « ouï-dire » - la célèbre fides ex auditu ${ }^{2}$. L'Épître aux Éphésiens $(2,8)$ dit, pour sa part, que la foi ne vient pas de nous, mais qu'elle est, littéralement, un «don de Dieu ». Ces textes sont un peu déroutants pour nos oreilles ou nos ornières modernes, mais ce n'est peut-être pas la moindre de leurs qualités.

Afin de développer cette altérité, peut-être irréductible, entre la foi (ou la croyance) et le savoir, je partirai d'une devise qui est, tout à fait par hasard, celle de l'Université de Montréal: "Fide splendet et scientia» ( Elle brille [splendeo: resplendir, briller, «exceller »...] par la foi et par la science»). En tant que devise, c'est elle qui doit présider à tout ce que nous faisons, à tout ce que nous sommes à l'université, même si on n'en prend pas conscience. C'est une excellente chose que les universités aient de telles devises, car elles peuvent peut-être nous aider à comprendre ce qui nous rassemble tous, malgré tout, dans un monde où le savoir tend à se spécialiser à l'extrême (devenant alors tout le contraire d'un savoir).

Et si l'on comprend mal le latin, le petit logo de l'Université de Montréal nous en donne une petite illustration, en mode de bande dessinée: audessus de la devise, on aperçoit un château situé sur une montagne, avec deux tours et, comme si l'on n'avait pas encore assez compris, deux petites étoiles qui resplendissent au-dessus de chacune des tours. Certains diront que le kitsch est total: deux étoiles qui brillent, deux tours, la foi et la science. On pourrait très facilement y voir une rémanence du passé de l'université, celui d'une époque - révolue bien sûr (nous sommes, comme chacun sait, les premiers à tout comprendre) - où l'université se tenait encore sous la férule de l'Église et avait aussi pour mission de transmettre une fides. Aujourd'hui, la devise de l'Université de Montréal pourrait donc être: «Splendet scientia» («Elle resplendit par la science»). Et la dernière fois que j'ai regardé, il n'y avait effectivement qu'une seule tour sur le Mont

2. Voir Thomas d'Aquin, Summa theologica, 2a-2ae, qu. 4, art. 8 (1941, 175). Voir aussi la «définition" de la foi proposée par Thomas: "Si quis ergo in forman definitionis hujusmodi verba reducere velit [donc seulement si on y tient!], potest dicere quod fides est habitus mentis, qua inchoatur vita ceterna in nobis, faciens intellectum assentire non apparentibus. " ( "Si quelqu'un veut ramener ces mots à la forme d'une définition, on peut dire que la foi est une disposition de l'esprit par laquelle commence en nous la vie éternelle et qui amène l'entendement à donner son assentiment à des choses qui n'apparaissent pas. » qu. 4, art. 2 [1941, 141]; nous traduisons) 
Royal. Il serait un peu banal, dira-t-on, pour une université, de dire qu'elle brille par la «science». Mais le fait est qu'il y a bel et bien des universités dont les devises expriment de tels truismes. Pensons, par exemple, à l'Université Harvard, dont la devise porte seulement «Veritas », sans verbe, sans adjectif, sans cas : vérité!

Notre devise a quelque chose d'un peu plus palpitant. Elle est l'expression d'un ménage à deux, où l'on pourrait dire, aujourd'hui, que c'est la science qui irradie le plus. Mais il n'empêche, c'est la fides qui se trouve nommée en premier lieu, voire sur elle que porte l'accent ( «Par la foi, elle resplendit, mais secondairement aussi par la science»). Qu'est-ce que la fides? Inépuisable question, déjà discutée tout au long de ce colloque, mais à laquelle il est difficile de répondre, et pour une raison de fond: toute explication de ce qu'est la foi la transformerait nécessairement en autre chose, en une explication rationnelle, par exemple. Mais alors, la foi n'est justement plus de la foi, mais une explication rationnelle ou de la science. C'est pourquoi il est si difficile de dire ce qu'elle est, car on la traduit alors en un langage qui n'est justement pas le sien.

Parlons peut-être des mots, puisqu'il vient d'être question du langage. Lorsque l'on parle de science et de foi, il va de soi que l'on compare deux termes grecs, "epistèmè » et "pistis", qui ont acquis pour nous un sens assez différent de celui qui était le leur chez les Grecs de l'Antiquité. On peut le voir dans le fait que l'opposition de l'epistèmè et de la pistis (ou, en latin, de la scientia et de la fides) a déjà été marquée par le père de la métaphysique, Platon, dont on sait l'influence plus que déterminante sur la spiritualité occidentale et sur les termes que nous utilisons pour en parler - influence si puissante d'ailleurs qu'elle est à peine perceptible. La distinction la plus proprement platonicienne est, bien sûr, celle de la doxa et de l'epistèmè, c'est-à-dire de l'opinion et du savoir. Mais au sixième livre de sa République, Platon distingue nettement l'epistèmè de la pistis. Si «epistèmè»" peut encore être rendu par «science", il va de soi que l'on ne peut pas du tout traduire «pistis» par «foi», en tout cas au sens religieux du terme. Par "pistis », Platon entend plutôt une conviction qui porte sur les réalités sensibles. Mon collègue Yvon Lafrance a d'ailleurs fort heureusement traduit «pistis» par le terme (un peu hégélien) de «certitude sensible ${ }^{3}$ (1994, 402),

3. «L'expression renvoie à la situation épistémique de l'homme ordinaire dont la connaissance repose sur les choses visibles et sensibles. [...] Ce sera donc le rôle du dialecticien de démasquer, pour ainsi dire, cette certitude sensible pour la déclarer "faillible" " (Lafrance 1994, 402). 
qui est celle du commun des mortels qui tient les réalités sensibles pour des entités dernières. C'est que, pour Platon, cette certitude n'est rien de plus qu'une simple conviction (les verbes "peithô » et "peithomai », qui ont la même racine que «pistis", veulent respectivement dire «convaincre» et "être persuadé de »), conviction qui correspond à un niveau très inférieur du savoir, parce que limité au monde sensible.

Lorsque Platon lui oppose l'epistèmè, ou la science, il pense à un savoir supérieur, dont l'excellence tient au fait qu'il ne repose pas sur l'expérience, mais sur des réalités idéelles. L'opposition entre la pistis et l'epistèmè a donc chez Platon une signification qui va un peu dans un sens contraire au sens que nous lui donnons. C'est que, pour nous, la science a d'abord affaire au monde sensible (en tant que "science de l'expérience»), alors que c'est la pistis, la «foi », qui aspire, dans une certaine mesure, au suprasensible. Chez Platon, c'est un peu le contraire: l'epistèmè est tournée "vers le haut» et la pistis «vers le bas".

Mais de Platon jusqu'à nous, il y a, à l'évidence, une continuité, qui tient à la supériorité ou au prestige que nous reconnaissons toujours au savoir scientifique. Son modèle de ce qu'est l'épistèmè ou la science, Platon l'empruntait aux mathématiques qui tirent des conclusions rigoureuses à partir de principes premiers que l'esprit peut apercevoir. C'est un savoir qui donne lieu à une réelle prise et même à une maîtrise ( «epistamai » veut, en effet, dire «je m'y connais », mais aussi «je suis capable de»). Ce type de savoir se distingue de l'opinion (doxa), qui ne dérive pas vraiment de principes supérieurs et dont l'acquisition n'a pas la rigueur de la connaissance mathématique. Aristote a assez largement repris cette conception de la science, dans ses Seconds Analytiques, en associant l'idée de science à un savoir qui peut faire l'objet d'une démonstration logique. Cette conception de la science s'est maintenue tout au long du Moyen Âge et bien au-delà (on la retrouve encore chez des auteurs comme Kant et Fichte ${ }^{4}$ ). Assurément, notre conception de la science n'est plus tout à fait celle de Platon, d'Aristote ou de Kant, mais nous continuons à y voir un type de connaissance plus rigoureux que celui de l'opinion (ou de la pistis) parce que susceptible d'être fondé.

Le passage de la pistis platonicienne à la notion de foi est beaucoup plus complexe. Un lien entre les deux est cependant donné par l'idée de

4. Qui ajouteront même cette exigence que la science constitue un tout systématique. Kant dira ainsi qu'une connaissance est une science lorsqu'elle forme non un agrégat de connaissances, mais un système, c'est-à-dire «l'unité de diverses connaissances sous une Idée» (Kant A 832/B 860 [2001, 674]). 
«conviction» ou de «persuasion". Nous entendons généralement par là une croyance religieuse, ce qui n'était pas du tout le cas des Grecs. Ces derniers, ceux de la période classique en tout cas, n'avaient pas vraiment de nom pour décrire ce que nous appelons la «foi » religieuse, sauf peut-être celui de "piété » ( "eusebeia»), mais celle-ci se manifeste davantage par des signes extérieurs que par une conviction intérieure. Cela est très curieux pour nous et nous rend assez incompréhensible ce que pouvait être leur «expérience religieuse».

C'est saint Paul qui se sert du terme "pistis » pour exprimer ce que nous rendons par «foi». Comme Rudolf Bultmann l'a montré, dans une étude classique (Bultmann et Weiser 1976), quoiqu'un peu ancienne (parution originale en 1959), sur la notion de pistis, saint Paul s'inspirait sans doute de l'idée, développée par la philosophie hellénistique et stoïcienne, selon laquelle la morale (et, par extension, le rapport au divin) relevait de la conviction personnelle. Saint Paul lui a aussi reconnu un prestige inouï (qu'elle n'avait pas du tout chez Platon) en disant qu'elle était plus essentielle que la science elle-même: n'est-il pas allé dire aux Corinthiens que Dieu avait frappé de folie la sagesse rationnelle de ce monde (1Co 1,20), dans un texte célèbre qu'un penseur comme Martin Heidegger n'hésitera pas à remettre sous le nez des théologiens qui cherchent à développer une conception rationnelle de la foi ? Je cite le texte de Heidegger, qui se trouve dans l'introduction qu'il a rédigée en 1949 à sa conférence "Qu'est-ce que la métaphysique ? " : «est-ce que la théologie chrétienne pourra enfin se décider à prendre au sérieux cette parole de l'Apôtre, c'est-à-dire prendre au sérieux ce qu'il a dit à propos de la philosophie comme d'une folie? » (1968, 41 [GA, 9, 374])

Toute réflexion sur la foi, le croire ou la croyance se tient dans l'ombre de cet avertissement de l'apôtre Paul, répercuté par Heidegger: n’y a-t-il pas un abîme entre la foi et la science, la fides et la scientia? Toute réflexion sur la foi n'est-elle pas radicalement différente de son objet et dès lors, fatalement, à côté de lui ?

Cette limitation n'a pas empêché, bien sûr, la tradition occidentale, judéo-islamo-chrétienne, de réfléchir «sur» la foi. Si je parle de cette tradition, ce n'est pas par ethnocentrisme, mais parce que je pense que cette tradition a sans doute été la seule à avoir expressément médité sur le sens de la foi. Des auteurs comme saint Grégoire, Augustin, Anselme, Thomas d'Aquin, Schleiermacher et Kant (et des quantités d'autres) ont, en effet, tenté de 
nous dire, et de manière très différenciée, ce qu'était la «foi ». Je ne sais pas s'il y a quelque chose d'équivalent à ce type de réflexion dans les autres cultures. Je ne le crois pas. On peut évidemment se demander si cette conception de la foi, cette «théorie de la foi », si j'ose dire, n'a pas affecté les conceptions que l'on se fait des croyances dans les autres cultures. J'évoquerai, rapidement, deux manières dont cela pourrait être le cas.

1) Le terme "pistis » inscrit, durablement, irrémédiablement, la «foi », la « croyance » dans l'ordre des convictions (peitô, peithomai), ordre essentiellement rhétorique qui présuppose que l'on a été persuadé ou convaincu — donc par des raisons ou des arguments — de quelque chose. Mais n'estce pas déjà une visée trop intellectualiste, mais aussi trop rhétorique de la foi ? Il est clair qu'elle porte la marque de la philosophie grecque, pour laquelle ce type de foi sera nécessairement inférieur au savoir scientifique, qui fait, lui, l'objet d'une démonstration logique. 2) Lorsque la tradition chrétienne pense la foi, considérons Thomas d'Aquin par exemple, elle la comprend tout naturellement comme une virtus, une vertu. Le terme vient encore une fois du grec, "arètè », que l'on peut aussi traduire par "excellence». Terme difficile à comprendre pour nous, parce que la vertu n'est plus pour nous qu'une affaire morale ou éthique, et encore (notre jargon éthique contemporain est d'une pauvreté inouie: on a toujours le sentiment - et cela donne des frissons - de lire des manuels d'économie). Aristote distinguait, comme chacun sait, les vertus intellectuelles (dianoétiques) des vertus pratiques (ou proprement éthiques). Or, la foi a spontanément été comprise comme une "vertu » par saint Thomas, pour lequel la "science » est elle-même une vertu (intellectuelle), comme chez Aristote. Il s'agit, bien sûr, de la première des trois vertus théologales (la foi, la charité et l'espérance, suivant une trinité qui remonte aussi à saint Paul). Mais encore ici, l'horizon grec restait déterminant puisqu'il pensait la vertu comme une excellence. Va-t-il de soi que la foi soit une vertu ou une excellence? N'estelle pas aussi, je dirai pourquoi à la fin, une absence de vertu?

Il a surtout été question jusqu'ici de la foi et très peu de la croyance (que certaines langues ne distinguent pas toujours; c'est le cas de l'allemand notamment, qui parle dans les deux cas de "Glaube»). Faut-il distinguer la foi religieuse des autres croyances qui nous habitent?

Augustin est de ceux qui ne se formalisaient aucunement de la parenté entre la foi et les croyances les plus banales. Il n'éprouvait aucun scrupule à inscrire la foi dans la continuité de nos croyances de vie. Dans son De 
utilitate credendi ${ }^{5}$, Augustin dit qu'il n'est pas de vie sans croyance, sans ce que l'on pourrait appeler une foi vitale et élémentaire. Quand un ami nous dit quelque chose, nous le croyons spontanément, remarque-t-il' ${ }^{6}$. Nous n'attendons pas de voir si cela est démontré (même si cela arrive parfois dans les rencontres amicales: attendez, je vais vérifier si...). Sinon, il n’y aurait jamais d'amitié. C'est de la même manière, souligne Augustin, que les enfants apprennent de leurs parents et les élèves de leurs maîtres, parce qu'ils leur font confiance. On pourrait même dire - et Augustin le disait, bien avant David Hume - que quelque chose de cette foi se retrouve au cœur de la science elle-même: on fait confiance, on a foi en ce que dit la science ou le scientifique. Si l'on reconnaît qu'une démonstration est rationnelle, c'est qu'on y croit (comme on croit au témoignage de ses propres yeux).

On pourrait aller plus loin et dire que le grand avantage de la foi, sur la science, est de se savoir justement «foi ». C’est ce qui a déjà amené un de mes professeurs, Josef Simon, philosophe, à dire que la «théologie » était peut-être la science la plus réfléchie, voire la plus avancée qui soit, au sens où elle savait, elle, qu'elle reposait sur la foi, alors que les autres sciences l'avaient un peu oublié (voir Simon 1977). Avec Gianni Vattimo, on pourrait aussi ajouter qu'elle est la seule à savoir qu'elle repose sur un ouï-dire, sur une fides ex auditu. Mais c'est aussi le cas de toutes les sciences'. Vous connaissez une science qui ne repose pas, dans une certaine mesure, sur le ouï-dire ? La théologie est cependant la seule à avoir les instruments permettant de le penser. C'est à ce titre, peut-on dire, que la théologie reste et doit rester, dans toute université, la faculté supérieure.

Venant de la faculté inférieure, celle de philosophie, la seule chose qui puisse m'intéresser ici est le rapport entre la foi et la science. Augustin nous invite à comprendre qu'il y a, en un certain sens, une priorité de la foi sur la science, en disant qu'il n'est pas de vie humaine ni même de savoir sans elle. Augustin le disait, bien sûr, dans un contexte particulier, qui n'est plus tout à fait le nôtre. Des essais comme le De utilitate credendi ou le De vera religione, voire les Confessions elles-mêmes, ont été écrits pour répondre aux

5. On dit souvent, depuis Hannah Arendt, qu'Augustin aurait placé le frui bien au-dessus de l'uti. Mais Dieu qu'il prêtait une très haute dignité à l'utile dans cet écrit !

6. De l'utilité de croire, X,24 (Augustin 1951, 265). Voir aussi le De fide rerum quoe non videntur [La foi aux choses qu'on ne voit pas], I,2 (1951, 312s.). Si, y note Augustin, on supprimait cette foi des relations humaines, une horrible confusion s'en suivrait — «orrenda confusio subsequatur» (II,4 [1951, 316]).

7. L'idée revient souvent dans ses derniers écrits. Voir par exemple Vattimo 2002, 28. 
manichéens, qui étaient les «rationalistes » de l'époque, semble-t-il, parce qu'ils prétendaient qu'avant de croire, il fallait déjà comprendre. Augustin parlait en connaissance de cause, puisqu'il a lui-même été manichéen, pendant neuf ans, et justement parce qu'il hésitait à se vouer à la religion catholique, dont il ne comprenait pas toutes les raisons. Et Augustin l'a fait en rappelant qu'il nous est justement impossible de connaître les raisons de toutes choses ${ }^{8}$.

Ceci m'amène à ma conclusion, sur les rapports de la foi et de la science. On a vu que la devise de l'Université de Montréal conjuguait la foi et la science, en leur conférant une certaine luminosité, qui rend toutes choses visibles, mais sans s'offrir elle-même à une vision. La science est un «savoir » qui assure une certaine maîtrise sur le réel: le verbe grec "epistamai ", d'où vient le terme «science» («epistèmè»), exprime d'ailleurs une certaine habileté, une capacité, un « je maîtrise... » (parce que j’en connais les principes). La foi procède, je crois, d'une autre expérience, mais qui la précède. Elle est moins l'expression d'un « je m'y connais », « je maîtrise... » (« epistamai ») qu'une façon de dire «je ne m’y connais pas, je ne maîtrise pas...». La foi est ainsi une certaine forme d'humilité, de pudeur, marquant une certaine réserve, une retenue là où la science apparaît plus impudique, sinon indécente, en tout cas très présomptueuse. Mais c'est un non-savoir qui reste présupposé, quoique oublié, en toute forme de savoir. Fide splendet et scientia. En ce sens, la foi (ou la croyance) est moins un savoir qu'un nonsavoir, moins un «je sais " qu'un « je ne sais pas », moins une vertu ou une excellence qu'une absence de vertu, moins une affirmation qu'un silence. Et dans la cacophonie de notre monde, il se pourrait effectivement que ce silence soit un don de Dieu.

\section{Références}

Augustin (1951), Euvres de saint Augustin, t. VIII : La foi chrétienne / texte de l'édition bénédictine, introductions, traduction et notes par J. Pegon, Paris, Desclée de Brouwer (Bibliothèque augustinienne).

Bultmann, R. et A. Weiser (1976) [allemand 1959], Foi / trad. par É. de Peyer, Genève, Labor et Fides. Cette traduction renvoie à l'article original

8. Augustin, op. cit., XII,26 (p. 272): multa possunt afferri, quibus ostendatur nibil omnino humance societatis incolumne remanere, si nibil credere statuerimus, quod non possumus tenere perceptum. 
"Pisteuô", dans Theologisches Wörterbuch zum Neuen Testament, t. VI, Stuttgart, Kohlhammer, 1959, p. 174-230.

Heidegger, M. (1968) [allemand 1949], Questions I / trad. par R. Munier, Paris, Gallimard (Classiques de la philosophie).

KANT, E. $\left(2001^{2}\right)$ [1997, allemand $1787^{2}$, 1781], Critique de la raison pure / traduction, présentation et notes par A. Renaut, Paris, Flammarion (Garnier Flammarion 1142).

Lafrance, Y. (1994), Pour interpréter Platon, t. II : La ligne en République VI, 509d-511e. Le texte et son histoire, Montréal, Bellarmin (Noêsis).

Simon, J. (1977), "Zur philosophischen Ortsbestimmung theologischer Wissenschaft von ihrem Gegenstand her ", Tübinger Theologische Quartalschrift, 157, p. 204-207.

Thomas D'Aquin (1941) [latin 1266-1272], Somme théologique. La foi. 2a-2ae. Questions 1-16 / trad. par R. Bernard, Paris / Tournai / Rome, Revue des Jeunes, t. I (questions 1-7).

Vattimo, G. (2002), "The Age of Interpretation", dans A. Wiercinski, dir., Between the Human and the Divine. Philosophical and Theological Hermeneutics, Toronto, The Hermeneutic Press.

\section{Résumé}

En partant d'une réflexion sur la devise de l'Université de Montréal, "C'est par la foi et la science qu'elle resplendit ", cet article se penche sur l'altérité fondamentale de la foi par rapport à la science, voire sur sa priorité. Il se demande si la science, dans la mesure où elle doit se tenir à une certaine distance de son objet, est en mesure de comprendre quelque chose à l'expérience de la foi. À la différence de la science, la foi est peut-être moins une forme de maîtrise que de dépossession, moins un savoir qu'un non-savoir.

\section{Abstract}

Starting with a reflection on the motto of the University of Montreal, "Through faith and science it shines", this article focuses on the fundamental alterity of faith vis-à-vis science, if not its priority with regard to science. It asks whether science, to the extent that it must adopt a certain distance toward its object, is able to understand the experience of faith as such. As opposed to science, faith is perhaps less a form of mastery than of depossession, less a form of knowledge than the absence thereof. 